\title{
GERENCIA Y ESTRUCTURA DE COSTOS EN PROYECTOS DE CONSTRUCCIÓN DE POZOS
}

\author{
Julio Macías Alvarenga
}

E-Mail: maciassa@racsa.co.cr

\begin{abstract}
Management and life cycle concepts can be applied to development projects (global project) where water wells are required. The construction of wells can be visualized as a subproject in the global project. Two phases are recognized in this subproject: feasibility and installation. During both phases, the hydrogeological consultant plays an important role.

This document summarizes a series of ideas about the role consultants play or should play in this type of projects. No new techniques or concepts are presented, but an application of the current knowledge in this field. A definition of costs and a guide to prepare a cost's structure is presented. This structure can be used by the consultant and also by the investor and its economical-financial team, to choose the optimal alternative of a well with the lowest cost . A real case example is developed..
\end{abstract}

RESUMEN: En los proyectos de desarrollo (proyecto global) donde se requiere de la captación de agua subterránea a través de pozos, pueden aplicarse los conceptos de gerencia y ciclo de vida del proyecto. En este sentido, la construcción de un pozo puede considerarse un sub-proyecto del proyecto global y por lo tanto estar sujeto también a la aplicación del enfoque gerencial. De esta manera, pueden reconocerse dos etapas, la formulación y la ejecución. En ambas el consultor en hidrogeología juega un papel relevante.

En este documento se resume una serie de ideas y reflexiones sobre el papel general que juega ó debe jugar el consultor en hidrogeología. No pretende crear nuevas técnicas o conocimiento sobre el tema, sino, aplicar los ya existentes. Se presenta un esquema de definición de costos y una guía para que el consultor elabore una estructura de costos que sea útil al inversionista y su equipo de evaluación económico- financiero, y que permita también la selección de la alternativa de pozo con menor costo posible. Un ejemplo de aplicación se desarrolla para un caso real.

\section{INTRODUCCIÓN}

Las técnicas de administración de proyectos de desarrollo han sido ampliamente investigadas y desarrolladas por diversos autores ( $\mathrm{Sa}-$ pag \& Sapag 1997, Rosales 2000, ICAP 2000). El objetivo final perseguido a través del empleo de estas técnicas es, en general, garantizar que los proyectos sean formulados y ejecutados de la mejor manera a fin de obtener resultados dentro del tiempo, costo y calidad estipulados.
Estas técnicas reconocen, entre otras, dos grandes etapas en el ciclo de vida del proyecto: la formulación y la ejecución.

La teoría de la formulación de proyectos, nos permite definir el producto, requisitos técnicos e ingenieriles, tiempo de duración, prever costos de inversión y operación y, además, permite realizar diseños preliminares o definitivos. Los resultados de esta etapa de formulación definen el grado de viabilidad y factibilidad con que cuenta un proyecto para su ejecución. Esta 
información es de vital importancia para el inversionista en la toma de decisiones sobre continuar con el desarrollo del mismo.

Una vez demostrada la viabilidad y factibilidad, se prosigue con la realización. Esta etapa de ejecución es, cuando se lleva a la práctica el proyecto, cuando se ejecutan las obras o se desarrolla las actividades proyectadas. Durante esta etapa, las obras se realizan de acuerdo con un cronograma de actividades, resultado de la programación, planeamiento y un esquema organizativo donde se definen las responsabilidades y flujos de información. Los resultados de la etapa de ejecución deberán coincidir con aquellos previstos dentro de la etapa de formulación. Para tal efecto, es importante realizar una tarea de control y seguimiento durante la ejecución. Esta labor garantiza que las actividades sean ejecutadas dentro del plazo establecido, con el costo presupuestado y con la calidad requerida.

El buen resultado de los proyectos de desarrollo garantiza que el bienestar económico aumente para toda la sociedad. Esto solo se conseguirá a través de proyectos rentables y altamente productivos. Debido a este impacto social directo, es importante que los profesionales involucrados en la formulación y ejecución cuenten con el conocimiento técnico idóneo y con el dominio de las herramientas gerenciales para realizar estas labores.

Dentro de este grupo de profesionales que juega un papel importante en el desarrollo de los proyectos, se encuentran los dedicados a la actividades geológicas (geotécnia, hidrogeología, impacto ambiental, etc.) En cada una de estas áreas se participa en proyectos donde es posible reconocer una etapa previa de estudios básicos coincidente con la formulación, y otra etapa de inspección durante la construcción coincidente con el control y seguimiento en la fase de ejecución.

El aprovechamiento del agua subterránea es parte importante de la viabilidad y factibilidad de muchos proyectos de desarrollo. Desde esta perspectiva, se puede reconocer dentro del proyecto global ideado por el inversionista (sea invernadero, urbanización, turísticos etc.), un subproyecto de construcción de pozo para aprove- chamiento de agua subterránea. En este proyecto de construcción de pozo pueden ser reconocidas las dos etapas previamente citadas. La etapa de estudios hidrogeológicos (formulación) y la etapa de inspección (control y seguimiento). De esto se desprende, que son claramente aplicables todas aquellas técnicas gerenciales desarrolladas para proyectos de desarrollo. Así, el profesional de esta área debe jugar un papel activo y responsable en relación con ambas fases del proyecto.

Este documento tiene como objetivo, presentar una serie de ideas y reflexiones sobre el papel general que debe jugar el consultor en hidrogeología. No pretende crear nuevas técnicas o conocimiento sobre el tema, sino, aplicar los ya existentes. Particular detalle se presenta al esquema de definición de costos. Se presenta una guía para que el consultor elabore un esquema de costos que sea útil al inversionista y su equipo de evaluación económico- financiero, y que también permita la selección de la alternativa de pozo con menor costo posible.

Es importante resaltar que este artículo se centra en el papel real del consultor, entendiendo el término como el profesional responsable que guía y orienta de manera objetiva y apegada al mejor saber y entender, al inversionista de tal forma que este tome la decisión con la información más completa posible con el presupuesto disponible.

A continuación se presentan los aspectos relacionados con las fases de formulación y ejecución de proyectos de construcción de pozos para captación de agua subterránea.

\section{ETAPA DE FORMULACIÓN}

Durante la etapa de formulación es cuando se conceptualiza el producto, el tamaño, localización, requerimientos técnicos, de ingeniería y además se estima los costos de las alternativas de pozo que garantizan el abastecimiento del volumen demandado.

Para la formulación de un proyecto de construcción de pozo, deben considerarse los siguientes aspectos: técnicos, ambientales y económico-financieros. 


\section{Aspectos Técnicos}

Estos se enmarcan dentro de los estudios básicos. Como primer paso, el consultor deberá conocer la demanda real de agua por parte del proyecto global durante su vida útil, esto es, caracterizar el producto esperado como lo es el volumen requerido y la calidad del líquido. Esto requiere que el consultor se involucre con la idea y las exigencias del inversionista.

Durante la formulación técnica de alternativas de pozo, el consultor participa generalmente de los siguientes aspectos:

\section{Caracterización de las condiciones hi-} drogeológicas del sitio donde se desarrollará el proyecto. Esta caracterización se basa en la consulta de estudios anteriores, registro de pozos cercanos, ensayos de campo y experiencia propia del consultor. Es importante definir las propiedades hidráulicas del acuífero de la manera más realista posible. El éxito de los pasos siguientes dependerá de contar con información confiable.

Análisis de Alternativas: Basados en los requerimientos del proyecto (volumen diario) y conociendo las características hidráulicas del acuífero, se procede al análisis de alternativas de pozos con los cuales es posible extraer diariamente el volumen solicitado.

Para el análisis de alternativas se emplea la formulación existente para el tipo de acuífero en estudio (libre, confinado, otro). Se recomienda que en la medida de lo posible se emplee la formulación no lineal en régimen transiente, esto porque permite incorporar los efectos del flujo turbulento y, además, permite determinar los abatimientos requeridos y el tiempo necesario para obtener el volumen deseado.

De este análisis de alternativas se obtendrá diferentes configuraciones de diámetros de pozo, longitud total, tiempo de bombeo diario estimado, tipo de bomba, para los cuales se obtiene el volumen deseado. Para cada una de estas alternativas se determinan los costos asociados para la inversión y operación. Se escogerá como la alternativa óptima aquella, cuyos costos a valor presente resulten menores.
Diseño: A partir del resultado de la etapa anterior, se obtiene cuál es la configuración que garantiza el volumen requerido al menor costo posible. Seguidamente se procede al diseño final de la alternativa seleccionada. Esto es, definir el tipo de tubería a utilizar, la resistencia a la compresión requerida, el tipo de rejilla a emplear y, en caso de tuberías ranuradas, definir el patrón de estas.

\section{Aspectos Ambientales}

Los aspectos ambientales determinan en muchas ocasiones la viabilidad de los proyectos. Aunque esto forma parte del concepto global del mismo, es importante que el consultor cuente con el conocimiento para definir desde la etapa temprana de la formulación, si existen regulaciones ambientales que impidan la ejecución o que impongan modificaciones al concepto original. Los costos asociados con estas posibles modificaciones deberán considerarse dentro de la estructura de costos.

\section{Aspectos Económico-Financieros}

Dependiendo del tipo de proyecto, la rentabilidad será analizada desde el punto de vista económico o financiero. En cualquiera de los casos es importante que los costos asociados con los trabajos del proyecto de construcción de pozo sean incorporados al flujo de caja del proyecto global. Es norma incorporar estos costos según el siguiente formato:

Costos de Inversión: son todos aquellos requeridos para iniciar el funcionamiento del pozo.

Costos de operación: son todos aquellos requeridos a futuro para mantener el funcionamiento normal del pozo.

Sobre la estructura del costo se detallará más adelante.

El resultado final de la etapa de formulación es un informe de "consultoría" hidrogeológica, que indica el diseño preliminar del pozo recomendado, los costos asociados y, basado en la 
experiencia, el tiempo requerido para su construcción. Este informe debe contener todos aquellos aspectos a supervisar durante la etapa de ejecución como son: estratigrafía, parámetros hidráulicos por medio de un ensayo de bombeo, tipo de tuberías, distribución de ranuras, etc., esto con el objetivo de realizar un control y seguimiento del tiempo de ejecución, el costo incurrido y la calidad con la que el pozo se construye.

El informe de consultoría hidrogeológico debe indicar si el proyecto cuenta con viabilidad y factibilidad técnica, económica - financiera y ambiental.

\section{ETAPA DE EJECUCIÓN}

Esta etapa se inicia posterior a la finalización de la etapa de formulación, lapso durante el cual se realiza la evaluación económica o financiera del proyecto global y se concreta el financiamiento. Es durante la etapa de ejecución que se contrata la empresa perforadora, se perfora y arma el pozo, se coloca la bomba, se realizan todas las conexiones eléctricas pertinentes y se procede con el ensayo de bombeo para la aceptación del pozo.

La participación del consultor en esta etapa se centra en el control y seguimiento del planeamiento realizado durante la formulación. Esta labor se realiza por medio de la supervisión. Los aspectos normalmente supervisados para garantizar tiempo de ejecución, calidad y costo son:

\section{Corroborar las premisas de diseño}

Esto incluye la descripción de los materiales encontrados y su comparación respecto de los previstos en el estudio hidrogeológico o de formulación. Este control permite prever desviaciones en la duración del tiempo de perforación y posibles aumentos o reducciones de costos respecto de los previstos en caso de requerir modificaciones del diseño preliminar.

\section{Control de calidad}

El control de calidad se refiere a la supervisión del diámetro de perforación, del cumpli- miento de características especificadas para los materiales, distribución de las rejillas o ranuras, y el análisis del ensayo de bombeo. Con esto se pretende controlar que el pozo se comporte dentro del margen previsto y abastezca el volumen solicitado.

El fin último de la supervisión es garantizar que el pozo sea construido dentro del lapso de tiempo previsto, al costo estipulado o alternativamente con una variación razonable, y que permite el abastecimiento del volumen diario requerido durante el tiempo de vida del proyecto y con agua de calidad de acuerdo con su uso.

\section{ESTRUCTURA DE COSTOS}

Aunque por lo general los aspectos administrativos no son parte de la formación básica del consultor en hidrogeología, es importante que el profesional entienda su labor como un servicio de consultoría integral. Es este profesional quien debería suministrar esta información sobre costos para que sea incorporada al flujo de caja del proyecto global.

Es la norma diferenciar los costos en costo de inversión y costos de operación. Se presenta a continuación la estructura de composición de cada uno de estos costos:

\section{Costos de Inversión}

Se reconocen los siguientes:

Costos Intangibles: Corresponden con aquellos originado por la consultoría durante la formulación del proyecto y aquellos originados por la supervisión durante la etapa constructiva.

Costos Tangibles: Están determinados por el costo de aquellas actividades destinadas a la construcción y armado del pozo. Estos costos se determinan con base en el diseño previo e incluyen:

- Costo del pozo armado (perforación y tubería)

- Costo de bomba sumergible 
- Costo del cable sumergible

- Costo de tubería de salida

- Costo del sello de pozo

- Costo del panel de control completo

\section{Costos de Operación}

Corresponden con aquellos que se incurre durante la fase de producción del pozo para mantener su operación normal. Estos deberán calcularse para cada año del horizonte de evaluación e incluyen:

- $\quad$ Costos del consumo diario de energía, basada en las tarifas según Resolución del Regulador General publicada en la Gaceta del 13 de marzo de 2001 (estos costos deberán actualizarse según las tarifas vigentes)

- Costo del control de la calidad del agua en caso de requerirse.

- Costos de limpieza del pozo en caso de requerirse.

- $\quad$ Costos anuales por canon por concesión de permiso para aprovechamiento del agua según resolución $\mathrm{N}^{\circ} 266255$ - MINAE publicado en La Gaceta $N^{\circ} 18,27$ de Enero 1998.

Dependiendo del tipo de proyecto, se requerirá completar la lista anterior con otros costos ó depreciaciones. Una vez definida la estructura de costos para el proyecto específico, se podrá proceder de dos maneras:

Incorporar los datos obtenidos al flujo de caja global del proyecto, sin aplicar factores de descuento (es decir valor futuro). Esto es, incluir dentro del análisis económico - financiero del proyecto global, los costos de inversión y operación del proyecto de construcción de pozo.

- $\quad$ En el caso de realizar un análisis de alternativas para el proyecto de construcción de pozo, con el objetivo de definir la alternativa óptima, se podrá descontar los costos de operación a valor presente y realizar la suma total junto con los costos de inver- sión. El menor valor obtenido será aquel que cumple con el menor costo proyectado y que suple la demanda de agua solicitada, tomándose esta como la alternativa escogida.

\section{IMPLEMENTACIÓN COMPUTACIONAL}

El procedimiento de diseño y selección de la alternativa óptima, desde el punto de vista de costo y factibilidad técnica, puede ser automatizado para agilizar los cálculos. Esta automatización se puede efectuar a través del uso de lenguajes de programación ó por medio del uso de programas comerciales de hojas de cálculo.

El autor ha desarrollado una hoja electrónica de cálculo, con la cual es posible definir y comparar de manera automática diferentes opciones de pozo para un volumen requerido especificado. Con este procedimiento se define: profundidad de perforación, características de la bomba, cantidades de tubería y cable y además, se calcula el costo total de inversión y los costos operativos para cada alternativa. El uso de este tipo de herramienta computacional también permite la incorporación de celdas para obtener costos descontados y realizar comparaciones a valor presente.

\section{EJEMPLO DE APLICACIÓN}

A continuación se presenta un caso de aplicación del procedimiento de selección y de generación de la estructura de costos.

\section{Datos de Entrada}

Nombre de Proyecto Global: Invernadero para cultivo de Plantas Ornamentales

Objetivo: Construir un pozo de captación de agua subterránea para abastecer con $176 \mathrm{~m}^{3} \mathrm{de}$ agua diaria durante 6 meses al año.

\section{Aspectos Hidrogeológicos}

Tipo de Acuífero: Libre

Transmisividad: $11,8 \mathrm{~m}^{2} /$ día 
Rendimiento específico: 0,018

Profundidad del nivel estático: $16 \mathrm{~m}$

Tipo de análisis: Régimen transiente - Flujo darciano Formulación empleada (tomado de Pérez, 1995):

$$
S_{p}=\frac{Q}{4 \pi T d} \ln \left(\frac{2,246 \cdot t \cdot T d}{S y \cdot r_{p}^{2}}\right)
$$

donde:

$\mathrm{S}_{\mathrm{p}}=$ abatimiento en el pozo $(\mathrm{m})$

$\mathrm{Q}=$ caudal de bombeo $\left(\mathrm{m}^{3} / \mathrm{s}\right)$

$\mathrm{Td}=$ transmisividad $\left(\mathrm{m}^{2} / \mathrm{s}\right)$

$\mathrm{t}=$ tiempo de bombeo $(\mathrm{s})$

$\mathrm{r}_{\mathrm{p}}=$ radio del pozo $(\mathrm{m})$

Sy $=$ rendimiento específico (adimensional)

\section{Aspectos Técnicos}

Requerimiento diario: $176 \mathrm{~m}^{3}$

Tanque almacenamiento: localizado $10 \mathrm{~m}$ sobre el nivel del terreno

Pérdidas máximas en la tubería de bomba: $1 \mathrm{~m}$ Pérdidas máximas en la tubería del pozo al tanque de almacenamiento: $1 \mathrm{~m}$

Distancia de protección entre bomba sumergible y nivel dinámico: $5 \mathrm{~m}$ (PPB)

Eficiencia promedio de Bomba: $70 \%$

Tiempo de bombeo anual: 6 meses

Diámetro de perforación: 10 “*

Diámetro de tubería de revestimiento: 6" *

Tipo de tubería de revestimiento: PVC SDR 26

Tipo de rejilla: Ranurada PVC SDR 26

El pozo deberá penetrar el acuífero al menos dos veces el abatimiento teórico.

* Se considera solamente una opción, por efectos de espacio y, además, porque constituyen los valores típicos empleados en el mercado costarricense.

\section{Aspectos Financieros}

La evaluación para el costo de este proyecto se realiza en dólares estadounidenses USD\$.
Costos de inversión:

- Costo por metro de pozo armado: \$66

- Costo por metro de tubería de salida: \$2,53

- Costo de bomba y accesorios: más adelante se indica los valores

Costos de Operación:

- Costo de energía eléctrica: \$/ kwh 0,0803*

- Costo de canon anual de concesión: \$ 92,07**

Otros datos:

- Horizonte de evaluación: 5 años

- Tasa de descuento empleada: $12 \%$ (valor indicado por el equipo económico - financiero del proyecto global)

- Incremento anual en el costo de energía eléctrica: $5,64 \%$

- Tipo de cambio oficial al 27 de Febrero de 2002: Colones 347, 55

(precio venta: colones por cada dólar USD\$)

* Valor para consumos menores a $3000 \mathrm{kwh}$ por mes según tarifa T - 7 aprobada en Resolución del Regulador General del 13 de marzo del 2001. El cual corresponde con 27,9 Colones/kwh.

* Se considera como un valor constante durante todo el horizonte de evaluación aunque podría descontarse con la devaluación anual , para cada año de análisis. Dato según tabla B. 3 , Decreto $\mathrm{N}^{\circ} 26625$ - MINAE. El valor en colones corresponde con 32000 Colones/año.

\section{Análisis Técnico}

\section{Tiempo de Bombeo}

Con este análisis se pretende determinar el tiempo de bombeo teórico a partir de la ecuación 1 , para abatimientos específicos predeterminados y diferentes caudales, también predeterminados. Los valores de abatimiento predefinidos varían desde 5 hasta $30 \mathrm{~m}$, en incrementos de $2,5 \mathrm{~m}$; y los caudales oscilan entre 0,1 y 4,8 1/s con incrementos de $0,1 \mathrm{l} / \mathrm{s}$.

En algunos casos los valores de tiempo obtenidos son superiores a las $24 \mathrm{~h}$. Debido a que 
el análisis se realiza para requerimientos diarios, se ha establecido un valor máximo de bombeo de $24 \mathrm{~h}$, para efecto de determinar el volumen diario extraído.

En la figura 1 se presenta la relación gráfica particular entre el tiempo y el caudal de bombeo, para obtener un abatimiento teórico de $15 \mathrm{~m}$. De esta figura es claro, que para esta configuración puede mantenerse el bombeo durante $24 \mathrm{~h}$ para caudales entre 0,1 y $2,2 \mathrm{l} / \mathrm{s}$. A partir caudales de bombeo de 2,3 1/s, el tiempo para obtener un abatimiento de $15 \mathrm{~m}$ disminuye a una tasa elevada, hasta valores de $0,056 \mathrm{~h}$ para un caudal de $4,8 \mathrm{l} / \mathrm{s}$.

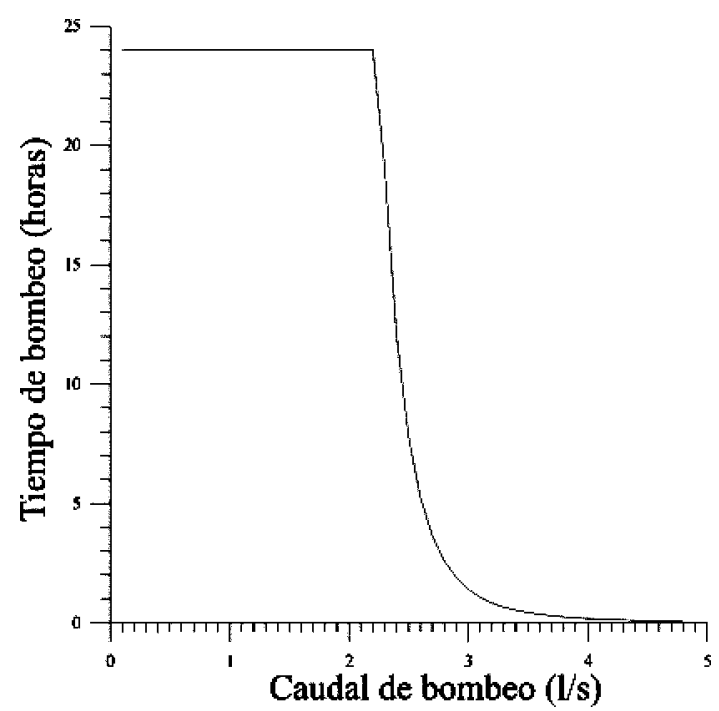

Fig. 1: Tiempo de bombeo teórico para diferentes condiciones de caudales de bombeo, para un abatimiento predefinido de $15 \mathrm{~m}$.

Este procedimiento de cálculo de tiempo de bombeo se puede realizar de manera automática e instantánea para todos las combinaciones de abatimiento teórico y caudal de bombeo estudiados a través del uso de una hoja electrónica de cálculo.

\section{Volumen extraído}

Una vez definidas las combinaciones posibles de tiempo y caudal de bombeo, se procede a determinar el volumen diario extraído $\left(\mathrm{en}^{3}\right)$, utilizando la siguientes relaciones:
Para tiempo de bombeo iguales o mayores a $24 \mathrm{~h}$ :

$$
\mathrm{V}=\mathrm{Q} * 24 * 3,6
$$

Para tiempo de bombeo menores a $24 \mathrm{~h}$ :

$$
\mathrm{V}=\mathrm{Q} * \mathrm{t}^{*} 3,6
$$

Donde:

$\mathrm{V}=$ volumen extraído $\left(\mathrm{m}^{3}\right)$

$\mathrm{Q}=\operatorname{caudal}(\mathrm{l} / \mathrm{s})$

$\mathrm{t}=$ tiempo durante el cual puede mantenerse el bombeo (h)

3,6 = factor de transformación $1 / \mathrm{s} \mathrm{a} \mathrm{m}^{3} / \mathrm{h}$

En la figura 2 se muestra la curva de variación del volumen diario extraído para diferentes condiciones de bombeo, para un abatimiento teórico de $15 \mathrm{~m}$. La curva en esta figura indica un comportamiento lineal creciente desde 0,10 a 2,20 1/s, región en la cual es posible mantener el bombeo durante $24 \mathrm{~h}$, como se presentó en la figura 1. El volumen máximo extraído de $190 \mathrm{~m}^{3} \mathrm{se}$ obtiene a un caudal de 2,20 1/s. Para caudales mayores a 2,20 1/s, el volumen extraído disminuye,

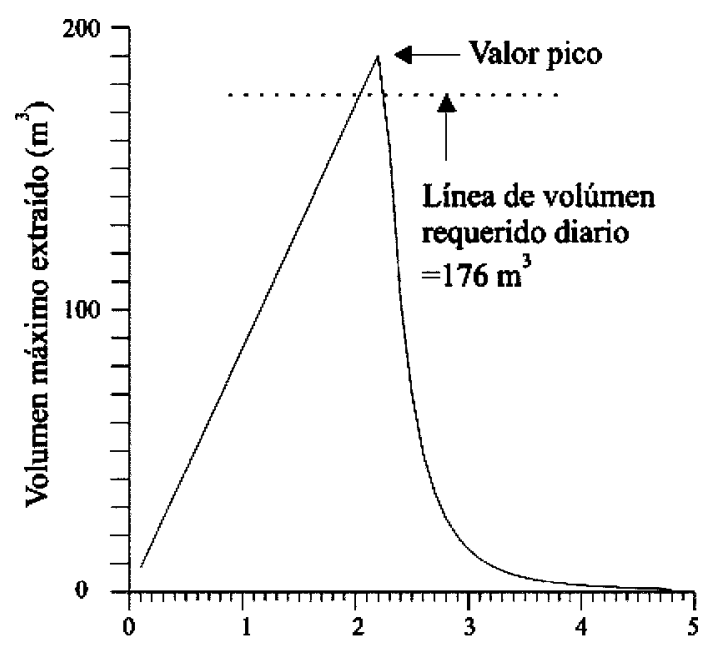

Caudal de bombeo $(1 / \mathrm{s})$

Fig. 2: Volumen diario máximo extraído para diferentes condiciones de caudal de bombeo, para un abatimiento predefinido de $15 \mathrm{~m}$. 
esto debido a que solo pueden mantenerse durante pocas horas.

En la misma figura 2 se ha trazado la línea de requerimento diario de volumen. Este valor es igualado o superado para caudales de bombeo entre 2,10 a 2,27 1/s. Del análisis de esta curva se desprende las siguientes condiciones de aceptación:

- Se aceptará como configuración factible o abatimiento teórico factible, aquel cuyo valor pico de volumen extraíble sea igual o superior al volumen requerido.

Solamente se considerará como caudales factibles de explotación aquellos, para los cuales el volumen extraído se localice entre el volumen requerido y el pico máximo extraíble del pozo. En el caso del pozo de la figura 2, solamente se considera como caudales explotables, aquellos comprendidos entre 2,10 y $2,27 \mathrm{l} / \mathrm{s}$.

- $\quad$ No se aceptará como caudales explotables aquellos que se localicen a la derecha del caudal que genera el volumen pico, aunque estos generen volúmenes mayores al volumen diario requerido. En el caso de la figura 2, aunque los caudales comprendidos entre 2,21 y 2,27 generan un volumen mayor o igual al requerido, se encuentra a la derecha del caudal que genera el volumen pico, esto es 2,20 1/s; por lo tanto no son aceptables. Esta condición se establece debido a que existe una disminución significativo del volumen extraído, para caudales superiores a aquel que genera el volumen pico. De esta manera se genera una seguridad adicional a la factibilidad técnica del proyecto.

Los cálculos realizados para la construcción de la figura 2 pueden ser computados de manera automática e instantánea para todas las combinaciones de abatimiento teórico y caudal de bombeo estudiados.

En las figuras 3 y 4 se muestra el conjunto de curvas generadas, tanto de tiempo de bombeo como de volumen extraído diario, para todas las diferentes condiciones de abatimiento teórico estudiadas. Las curvas de las figuras 1 y 2 corresponden con casos particulares de aquellos de la figura 3 y 4 .

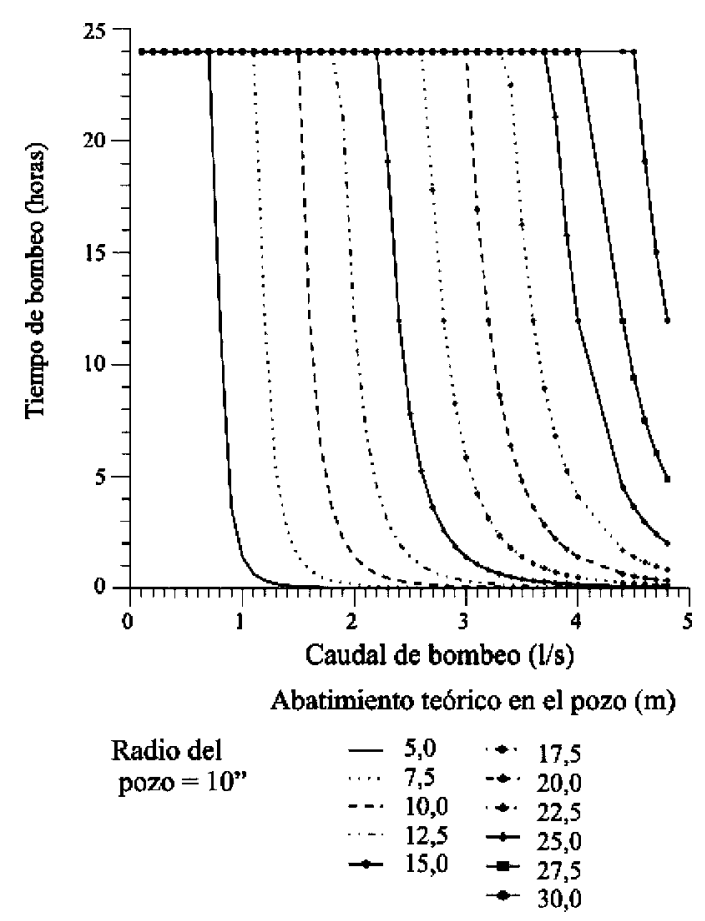

Fig. 3: Tiempo de bombeo teórico para diferentes caudales de bombeo en distintas condiciones de abatimiento predefinido en el acuífero.

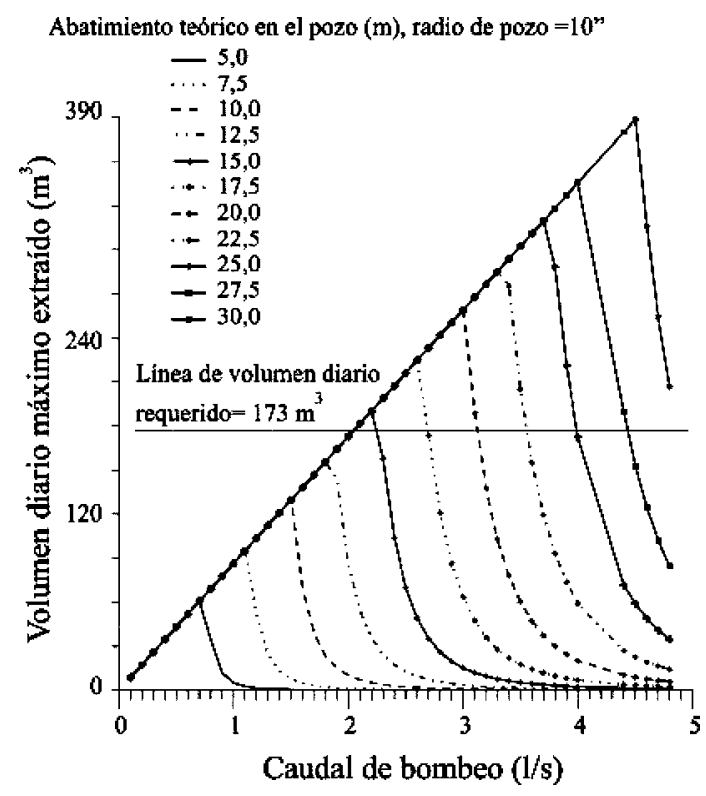

Fig. 4: Volumen diario máximo extraído para diferentes condiciones de caudal de bombeo, para abatimientos definidos. 
En la figura 4 se muestra además la línea de volumen requerido para el proyecto. Es claro de esta figura, que todas aquellas configuraciones de pozo donde sea posible obtener abatimientos mayores a $15 \mathrm{~m}$ serán factibles desde el punto de vista técnico pues todas ellas aportarán el volumen solicitado. Para todas estas opciones se aplican las tres condiciones de aceptación arriba planteadas.

\section{Profundidad del pozo}

La profundidad del pozo para cada configuración de abatimiento estará definida por, profundidad hasta el nivel de aguas + penetración requerida en el acuífero:

$$
\mathrm{PP}=\mathrm{PNE}+2 \mathrm{AT}
$$

Donde:

$\mathrm{PP}=$ Profundidad del pozo $(\mathrm{m})$

$\mathrm{PNE}=$ Profundidad del nivel estático $(\mathrm{m})$

$\mathrm{AT}=$ Abatimiento teórico predefinido $(\mathrm{m})$

Nota: Se estableció como requisito una penetración igual a dos veces el abatimiento teórico.

Al estudiar cada configuración de abatimiento, de manera indirecta se está analizando pozos con diferentes profundidades. Estos cálculos pueden ser realizados de manera automática por la hoja de cálculo electrónica.

\section{Bomba requerida}

La selección de la bomba se refiere a la determinación de la potencia y caudal necesario para cumplir con la demanda solicitada.

En relación con el caudal: Del análisis de la figura 4 se observa que para diferentes configuraciones de abatimiento, el volumen pico es variable. Este volumen pico se considera como el valor óptimo extraíble para un pozo con determinada profundidad. Como también se explicó anteriormente este volumen pico es obtenido para un caudal específico, el cual aumenta a medida que el pozo se torna más profundo puesto que se dispone de un abatimiento mayor. Con el uso de una hoja electrónica se determina de manera automática el valor de caudal que genera el volumen pico para cada una de las configuraciones de abatimiento estudiadas.

En relación con la potencia: La potencia de la bomba depende del caudal de trabajo y la elevación hasta la cual se desea levantar la columna de agua. Esta elevación está definida por:

$\mathrm{H}=\mathrm{ET}+\mathrm{PNE}+\mathrm{PPB}+\mathrm{AT}+\mathrm{PeTb}+\mathrm{PeTe}$

Donde

$\mathrm{H}=\quad$ Altura requerida para levantar la columna de agua $(\mathrm{m})$

ET $=\quad$ Elevación sobre el nivel del terreno del tanque de almacenamiento $(\mathrm{m})$

$\mathrm{PNE}=$ Profundidad del nivel estático $(\mathrm{m})$

$\mathrm{PPB}=$ Distancia de seguridad entre el nivel dinámico y la bomba $(\mathrm{m})$

$\mathrm{AT}=\quad$ Abatimiento teórico $(\mathrm{m})$

$\mathrm{PeTb}=$ Pérdidas en la tubería de salida de la bomba hasta la boca del pozo $(\mathrm{m})$

$\mathrm{PeTe}=$ Pérdidas en la tubería hacia el tanque de almacenamiento $(\mathrm{m})$

Una vez definida la altura $\mathrm{H}$, se determina la potencia requerida para el caudal de bombeo a través de la siguiente formulación:

$$
P o t=\frac{Q \cdot H \cdot 9,8}{E \cdot 100}
$$

Donde:

Pot $=$ Potencia $(\mathrm{kw})$

$\mathrm{Q}=\quad$ Caudal $(1 / \mathrm{s})$

$\mathrm{H}=\quad$ Elevación requerida $(\mathrm{m})$

$\mathrm{E}=\quad$ Eficiencia del motor de la bomba $(0,70$ para este ejemplo)

Los valores Pot pueden ser transformados a unidades hp, al ser divididos por 0,746. Al igual que la determinación de la profundidad del pozo, las especificaciones de los requerimientos de la bomba pueden ser calculados de manera automática por una hoja electrónica. 
En el cuadro 1 se presenta el resumen de los resultados obtenidos para las configuraciones técnicamente factibles. Esto es, todas aquellas profundidades de pozo y características de bomba, tiempo y caudal de bombeo que cumplen con la demanda de agua establecida de $176 \mathrm{~m}^{3}$ diarios.

\section{Análisis de Costos}

Para cada una de las opciones mostradas en el cuadro 1, se determina los costos asociados:

\section{Costos de Inversión}

En el cuadro 2 se presenta el desglose de los costos de inversión.

\section{Costos de Operación}

En los cuadros 3 y 4 se presenta el detalle de los costos de operación no descontados, los cuales en este caso, incluyen el consumo de energía y el canon por concesión de aprovechamiento del agua.

\section{Análisis de Resultados}

Como se indicó anteriormente, una vez definidos los costos para cada alternativa, se dispone de dos procedimientos de trabajo. El primero consiste en realizar la sumatoria de todos los costos a valor presente y definir la alternativa con menor costo. En el cuadro 5 se presenta el resumen de los costos descontados para cada alternativa. En la columna final se muestra también el costo total del pozo.

El segundo procedimiento consiste en que posterior a la selección de esta alternativa, se resume todos los costos sin descontar en una estructura de presentación de manera tal que permitan ser incluidos dentro del flujo de caja general del proyecto global.

\section{Cuadro 1}

Resumen de resultados para opciones técnicamente factibles

\begin{tabular}{cccccc}
\hline $\begin{array}{c}\text { Abatimiento } \\
\text { teórico } \\
(\mathrm{m})\end{array}$ & $\begin{array}{c}\text { Profundidad } \\
\text { requerida del pozo } \\
(\mathrm{m})\end{array}$ & Caudal óptimo & $\begin{array}{c}\text { Tiempo de } \\
\text { bombeo } \\
(\mathrm{h})\end{array}$ & $\begin{array}{c}\text { Potencia requerida } \\
\text { de la bomba } \\
(\mathrm{hp})\end{array}$ & $\begin{array}{c}\text { Profundidad de } \\
\text { colocación de bomba } \\
(\mathrm{m})\end{array}$ \\
\hline 15,0 & 44 & 2,2 & 22,2 & 1,9 & 34,0 \\
17,5 & 49 & 2,6 & 18,8 & 2,4 & 36,5 \\
20,0 & 54 & 3,0 & 16,3 & 2,9 & 39,0 \\
22,5 & 59 & 3,3 & 14,8 & 3,3 & 41,5 \\
25,0 & 64 & 3,7 & 13,2 & 3,9 & 46,0 \\
27,5 & 69 & 4,7 & 12,2 & 4,4 & 49,5 \\
30,0 & 74 & 4,5 & 10,9 & 5,2 & 49,0 \\
\hline
\end{tabular}

\section{Cuadro 2}

Resumen de costos de inversión para las alternativas técnicamente factibles

\begin{tabular}{ccccccc}
\hline $\begin{array}{c}\text { Profundidad } \\
\text { de pozo }\end{array}$ & $\begin{array}{c}\text { Longitud } \\
\text { total de } \\
\text { perforación } \\
(\mathrm{m})\end{array}$ & $\begin{array}{c}\text { Longitud } \\
\text { de tubería } \\
\text { de salida } \\
(\mathrm{m})\end{array}$ & $\begin{array}{c}\text { Costo total } \\
\text { de perforación } \\
\text { armado } \\
\text { USD\$ }\end{array}$ & $\begin{array}{c}\text { Costo de } \\
\text { tubería de } \\
\text { salida } \\
\text { USD\$ }\end{array}$ & $\begin{array}{c}\text { Costos de } \\
\text { bomba y } \\
\text { accesorios } \\
\text { USD\$ }\end{array}$ & $\begin{array}{c}\text { Costo } \\
\text { total } \\
\text { de inversión } \\
\text { USD\$ }\end{array}$ \\
\hline 44 & 44 & 34,0 & 2904 & 86,0 & 820 & 3810,0 \\
49 & 49 & 36,5 & 3234 & 92,3 & 900 & 4226,3 \\
54 & 54 & 39,0 & 3564 & 98,7 & 1000 & 4662,7 \\
59 & 59 & 41,5 & 3894 & 105,0 & 1000 & 4999,0 \\
64 & 64 & 44,0 & 4224 & 111,3 & 1300 & 5635,3 \\
69 & 69 & 46,5 & 4554 & 117,6 & 1400 & 6071,6 \\
74 & 74 & 49,0 & 4884 & 124,0 & 1600 & 6608,0 \\
\hline
\end{tabular}


Cuadro 3

Resumen de los costos de operación no descontados por consumo de energía

\begin{tabular}{|c|c|c|c|c|c|c|}
\hline $\begin{array}{c}\text { Profundidad } \\
\text { del pozo }\end{array}$ & $\begin{array}{c}\text { Consumo } \\
\text { diario de } \\
\text { energía } \\
\text { kwh }\end{array}$ & $\begin{array}{c}\text { Costo } \\
\text { consumo } \\
\text { energía } \\
\text { año } 1 \\
\text { USD\$ }\end{array}$ & $\begin{array}{c}\text { Costo } \\
\text { consumo } \\
\text { energía } \\
\text { año } 2 \\
\text { USD\$ }\end{array}$ & $\begin{array}{c}\text { Costos } \\
\text { consumo } \\
\text { energía } \\
\text { año } 3 \\
\text { USD\$ }\end{array}$ & $\begin{array}{c}\text { Costo } \\
\text { consumo } \\
\text { energía } \\
\text { año } 4 \\
\text { USD\$ }\end{array}$ & $\begin{array}{c}\text { Costo } \\
\text { consumo } \\
\text { energía } \\
\text { año } 5 \\
\text { USD\$ }\end{array}$ \\
\hline 44 & 31,484 & 454,51 & 480,14 & 505,78 & 531,41 & 557,05 \\
\hline 49 & 33,196 & 479,21 & 506,24 & 533,27 & 560,29 & 587,32 \\
\hline 54 & 34,907 & 503,91 & 532,33 & 560,75 & 589,17 & 617,60 \\
\hline 59 & 36,618 & 528,61 & 558,43 & 588,24 & 618,06 & 647,87 \\
\hline 64 & 38,329 & 553,32 & 584,52 & 615,73 & 646,94 & 678,14 \\
\hline 69 & 40,400 & 578,02 & 610,62 & 643,22 & 675,82 & 708,42 \\
\hline 74 & 41,751 & 602,72 & 636,71 & 670,71 & 704,70 & 738,69 \\
\hline
\end{tabular}

\section{Cuadro 4}

Resumen de costos de operación no descontados por canon de concesión de aprovechamiento

\begin{tabular}{cccccc}
\hline $\begin{array}{c}\text { Profundidad } \\
\text { del pozo } \\
(\mathrm{m})\end{array}$ & $\begin{array}{c}\text { Costos } \\
\text { USD\$ } \\
\text { año 1 }\end{array}$ & $\begin{array}{c}\text { Costos } \\
\text { USD\$ } \\
\text { año 2 }\end{array}$ & $\begin{array}{c}\text { Costos } \\
\text { USD\$ } \\
\text { año 3 }\end{array}$ & $\begin{array}{c}\text { Costos } \\
\text { USD\$ } \\
\text { año 4 }\end{array}$ & $\begin{array}{c}\text { Costos } \\
\text { USD\$ } \\
\text { Año 5 }\end{array}$ \\
\hline $\begin{array}{c}\text { Todas las } \\
\text { opciones } \\
\text { técnicamente } \\
\text { factibles }\end{array}$ & 92,07 & 92,07 & 92,07 & 92,07 & 92,07 \\
\hline
\end{tabular}

Cuadro 5

Costos totales descontados

\begin{tabular}{cccccccc}
\hline $\begin{array}{c}\text { Profundidad } \\
\text { del pozo } \\
(\mathrm{m})\end{array}$ & $\begin{array}{c}\text { Costo } \\
\text { inversión } \\
\text { USD\$ } \\
\text { año 0 }\end{array}$ & $\begin{array}{c}\text { Costo } \\
\text { operación } \\
\text { USD\$ } \\
\text { año 1 }\end{array}$ & $\begin{array}{c}\text { Costo } \\
\text { operación } \\
\text { USD\$ } \\
\text { año 2 }\end{array}$ & $\begin{array}{c}\text { Costo } \\
\text { operación } \\
\text { USD\$ } \\
\text { año 3 }\end{array}$ & $\begin{array}{c}\text { Costo } \\
\text { operación } \\
\text { USD\$ } \\
\text { año 4 }\end{array}$ & $\begin{array}{c}\text { Costo } \\
\text { operación } \\
\text { USD\$ } \\
\text { año 5 }\end{array}$ & $\begin{array}{c}\text { Costo } \\
\text { total } \\
\text { final } \\
\text { USD\$ }\end{array}$ \\
\hline 44 & 3810,0 & 497,88 & 464,97 & 433,40 & 403,26 & 374,60 & 5984,11 \\
49 & 4226,3 & 519,94 & 485,78 & 452,97 & 421,61 & 391,77 & 6498,37 \\
54 & 4662,7 & 541,99 & 506,58 & 472,53 & 439,96 & 408,95 & 7032,71 \\
59 & 4999,0 & 564,05 & 527,38 & 492,10 & 458,32 & 426,13 & 7466,98 \\
64 & 5635,3 & 586,10 & 548,18 & 511,66 & 476,67 & 443,31 & 8201,22 \\
69 & 6071,6 & 608,16 & 568,99 & 531,23 & 495,03 & 460,49 & 8735,50 \\
74 & 6608,0 & 630,21 & 589,79 & 550,79 & 513,38 & 477,67 & 9369,84 \\
\hline
\end{tabular}

Del cuadro 5 es claro que la opción con menor costo se consigue para un pozo con $44 \mathrm{~m}$ de profundidad, el cual requiere, según los datos del cuadro 1, una bomba con capacidad para 2,2 1/s y 1,9 HP de potencia.
Los costos asociados con esta alternativa pueden incorporarse al análisis financiero o económico general del proyecto, si son presentados en el formato mostrado en el cuadro 6. 
Cuadro 6

Esquema de costos (USD\$) para alternativa seleccionada (valores no descontados)

\begin{tabular}{ccccccc}
\hline Costo & Año 0 & Año 1 & Año 2 & Año 3 & Año 4 & Año 5 \\
\hline $\begin{array}{c}\text { Inversión } \\
\text { Intangible } \\
\text { Tangible } \\
\text { Operación }\end{array}$ & $*$ & & & & & \\
\hline
\end{tabular}

* Valor definido por el consultor de mutuo acuerdo con el inversionista.

\section{Diseño Final}

De esta forma, el diseño final preliminar del pozo que satisface los requerimientos del proyecto (176 m³ día) y, además, representa el menor costo posible, corresponde para un pozo con las siguientes características:

Profundidad total $=44 \mathrm{~m}$

Diámetro de perforación = 10 "

Diámetro de tubería de revestimiento $=6$ " PVC SDR 26

Rejilla tubería ranurada $=6$ " PVC SDR26

Sección ranurada a ser definida por el consultor. bomba recomendada $=4$ ", $2 \mathrm{hp}$, Caudal 2 1/s

Profundidad de colocación $=34 \mathrm{~m}$

Tiempo de bombeo previsto $=22 \mathrm{~h}$

Costo de inversión $=$ USD $\$ 3810+$ Costo consultor

\section{CONCLUSIONES}

La demanda creciente de competitividad en los proyectos de desarrollo, requieren que estos sean formulados y ejecutados de acuerdo con los estándares de las técnicas de alta gerencia. El consultor en hidrogeología, particularmente involucrado en el análisis y selección de alternativas para construcción de pozos para captación de agua subterránea, debe entender la importancia de la aplicación de estas técnicas y aprender cómo emplearlas en su labor de consultoría.

Aunque esto no forma parte estricta de los cursos de formación básica, es cada día más importante que esté familiarizado con este conoci- miento de manera tal que su labor de consultoría sea realizada de forma integral y que, además, le permita dominar la jerga y los elementos normalmente utilizados por los inversionistas para la toma de decisiones sobre la factibilidad y viabilidad de los proyectos.

En este artículo se han presentado reflexiones introductorias sobre las fases del proyecto y las actividades normalmente desarrolladas por estos consultores. Particular detalle se ha prestado al desarrollo de un ejemplo de aplicación de una metodología para la selección de alternativas. Como resultado final de este análisis se obtiene el costo óptimo de la alternativa que satisface la demanda de agua. Adicionalmente, se presenta una estructura de costos, la cual puede incluirse como parte integral del flujo de caja global, para la evaluación económica o financiera. Debido a su versatilidad y facilidad de implementación, las herramientas computacionales permiten la automatización de la selección de alternativas de manera ágil y confiable.

Aunque este documento no presenta técnicas o conocimientos nuevos, sino, más bien es el resultado de la aplicación de los ya conocidos, es el deseo del autor contribuir a mejorar el nivel de la consultoría en hidrogeología que se realiza en el país.

\section{REFERENCIAS}

PÉREZ FRANCO, D.,1995: La explotación del agua subterránea. Un nuevo enfoque. - 500 págs. Coedición MTI Ltda - Ed. Científico-Técnica, La Habana. 
SAPAG, N. \& SAPAG, R.,1997: Preparación y evaluación de proyectos. - [3ra ed.] 404 págs. McGraw-Hill, Bogotá.

ROSALES, P. R., 2000: Formulación y evaluación de proyectos.- [1ra reimpresión] 217 págs. Inst. Centroamericano Admin. Públ. San José.
INSTITUTO CENTROAMERICANO DE ADMINISTRACIÓN PÚBLICA, 2000: Guías para elaborar el diseño administrativo para la ejecución del proyecto. - 35 págs. Documento parte del curso de capacitación en alta gerencia de proyectos de desarrollo. 